\title{
Residual elastic strain due to laser shock peening: modelling by eigenstrain distribution
}

\author{
A M Korsunsky \\ Department of Engineering Science, University of Oxford, Parks Road, Oxford OX1 3PJ, UK. \\ email: alexander.korsunsky@eng.ox.ac.uk
}

The manuscript was received on 14 June 2005 and was accepted after revision for publication on 29 November 2005.

DOI: 10.1243/03093247JSA141

\begin{abstract}
High-energy synchrotron X-ray diffraction measurements of residual elastic strain were carried out in a thin slice parted off from a plate of titanium alloy that had been subjected to laser shock peening. The residual elastic strain varies with the distance from the laser shock peened surface, with high near-surface compressive strain changing to tensile strain in the middle of the sample, and then becoming compressive again on the opposite face. The measured residual elastic strain distribution was modelled using a distribution of laser shock induced eigenstrains near the surface, and the most likely eigenstrain profile was deduced using a variational matching procedure. The mathematical framework for this approach is presented and discussed, and the results of matching the predicted residual elastic strain distribution to the measurement are shown.
\end{abstract}

Keywords: data analysis, elasticity theory, residual elastic strain, synchrotron X-ray, monochromatic diffraction, energy-dispersive diffraction, strain mapping

\section{INTRODUCTION}

A companion article on high-energy synchrotron $\mathrm{X}$-ray diffraction measurement and interpretation of residual elastic strains in a laser shock-peened plate [1] presented the data that serves as the input for the present study.

Problems of evaluating the residual stress state in specimens and components arise frequently in connection with various engineering applications, such as the requirement to assess and minimize distortion occurring in manufacturing and processing, or the need to assess the effect of surface treatment on high cycle fatigue life (durability analysis). Process modelling of the sample or component deformation history offers one rational approach to the prediction of residual stress. However, in practice difficulties invariably arise in establishing the precise processing parameters (e.g. temperature fields, forces exerted by constraints) and in characterizing completely the dependence on the process conditions of the material properties (yield stress, hardening modulus, thermal conductivity). As a consequence, despite the complexity of the underlying constitutive equations, process models are often used primarily to provide qualitative understanding of the relationships between process conditions and physical parameters. Quantitative predictions of sophisticated process models can only be used after extensive validation against experimental measurements.

In this context, non-destructive experimental residual strain analysis provides a very useful tool in two important respects. Firstly, measurements of residual strains (and/or stress/distortion) provide a highly sensitive measure of the model's accuracy. Indeed, it is difficult to imagine a process model that would match correctly the spatial distribution of the components of residual elastic strain tensor, yet be significantly wrong in some other respect, e.g. thermal history. Secondly, in carefully assessed situations, measurements of residual elastic strains may provide an opportunity of introducing an intermediate reference state that may serve as the starting point for subsequent simulations of deformation. For example, if the state of residual strain is characterized well for a component that has undergone surface treatment by shot peening, then these data may serve as input for the model of subsequent non-linear 
deformation under cyclic loading conditions for the purpose of fatigue life prediction. It is particularly important to point out that suitable characterization in this context means the use of intrinsic parameters (such as plastic strain) rather than extrinsic parameters (dependent on the boundary conditions) such as stress.

Certain advantages of using plastic strain (or eigenstrain) as the basis for residual stress analysis can be identified. By the very definition of elastic deformation it cannot lead to the creation of residual stress: after removal of external forces any elastic object must return to precisely the same shape and dimension, i.e. the condition of zero elastic strain and hence stress. It is the introduction of permanent inelastic deformation (eigenstrain) that is responsible for residual stress. Additionally, eigenstrain distributions are more compact and more stable than residual stress distributions. As an illustration of this point consider an elastoplastic indentation of a plate that leads to some local permanent deformation. Numerical simulation readily shows that the residual stress state persists over much larger distances from the indent than the plastic strain. If the plate edge lies relatively closely to the indentation, then the resulting residual stress state is disturbed (modified) by its presence, unlike the plastic strain distribution. Finally, if the underlying plastic strain (eigenstrain) distribution is known, then the residual elastic strain and residual stress field can be found from the solution of an essentially elastic problem containing a perturbation.

The mathematical fundamentals of eigenstrain theory have been summarized in the book by Mura [2], where explicit analytical integral expressions are presented for elastic strain and stress fields due to known eigenstrain distributions. However, these expressions are only readily obtained for infinite or semi-infinite three-dimensional or two-dimensional problems. The application of these classical formulae to finite geometries requires significant computational efforts, since it is associated with the use of complex kernels that are themselves usually expressed in the form of integral transforms. The approach presented here, in contrast, relies on the simple bending formulation that gives rise to straightforward calculations that can be very readily accomplished, e.g. by using a spreadsheet.

When general complex geometries are considered the use of finite element formulations offers a tool that possesses sufficient flexibility. The presence of eigenstrain can be incorporated in these formulations through the use of nodal forces (e.g. see reference [3]) or the use of fictitious temperature fields and anisotropic thermal expansion tensors [4].

Whether analytical formulation or finite element framework is used, the task of matching the predicted elastic fields (i.e. the solution of the direct problem) to the observations remains paramount. The objective of the present article is to illustrate how the characterization of a state of residual elastic strain ought to be carried out in terms of the permanent plastic strain (or eigenstrain) by matching model predictions to the measurements. Simple bending theory formulation is used in the present case, since it allows the fundamental aspects of the approach to be readily clarified.

\section{DIRECT PROBLEM: DETERMINATION OF RESIDUAL ELASTIC STRAIN FROM A GIVEN EIGENSTRAIN}

Consider an elastic beam occupying the region $x_{\mathrm{L}}<x<x_{\mathrm{R}},-\infty<y<\infty$, and containing a distribution of eigenstrain $\varepsilon_{y y}^{*}=\varepsilon^{*}(x)$. The basic framework for evaluating the residual elastic strain (RES) distribution $e_{y y}(x)$ that arises in the beam has been presented in reference [5] and will only be reproduced here in brief to introduce some modifications to the previously published results.

The following statements provide the basis for the analysis.

1. Total strain in the beam is given by the sum of the elastic and inelastic strains (eigenstrains).

2. Following Kirchhoff's hypothesis of straight normals, it is assumed that material points originally lying on a line perpendicular to the beam axis remain on a straight line, i.e. any normal to the beam axis only undergoes rotation without distortion.

3. Hence displacements, and therefore total strain must vary linearly through the plate thickness, i.e. be given by

$$
\varepsilon=e+\varepsilon^{*}=a+\frac{b x}{h}
$$

where $h=x_{\mathrm{R}}-x_{\mathrm{L}}$ is the beam thickness. Here the parameter $a$ characterizes the amount of axial straining experienced by the beam and term $b$ characterizes the intensity of bending.

4. In the absence of external loading being applied, elastic strain $e$ presents an example of macroscopic residual elastic strain, such as that measured in a diffraction experiment. 
5. From equation (1), residual elastic strain is given by

$$
e=a+\frac{b x}{h}-\varepsilon^{*}(x)
$$

If the dependence of parameters $a$ and $b$ on the eigenstrain distribution $\varepsilon^{*}(x)$ is known, then the relationship between the residual elastic strain $e$ and the eigenstrain $e^{*}(x)$ is established.

6. It will be shown (below) that parameters $a$ and $b$ depend solely on two integral parameters, namely the zeroth and first moments of the eigenstrain distribution given by

$$
\Gamma=\frac{1}{h} \int_{x_{\mathrm{L}}}^{x_{\mathrm{R}}} \varepsilon^{*}(x) \mathrm{d} x, \quad \Gamma_{1}=\frac{1}{h^{2}} \int_{x_{\mathrm{L}}}^{x_{\mathrm{R}}} \varepsilon^{*}(x) x \mathrm{~d} x
$$

The relationship between parameters $a$ and $b$, on the one hand, and $\Gamma$ and $\Gamma_{1}$, on the other, is established using the requirements of force and moment balance across the beam, given by

$$
\begin{aligned}
& F=\int_{x_{\mathrm{L}}}^{x_{\mathrm{R}}}\left[a+\frac{b x}{h}-\varepsilon^{*}(x)\right] \mathrm{d} x=0 \\
& M=\int_{x_{\mathrm{L}}}^{x_{\mathrm{R}}}\left[a+\frac{b x}{h}-\varepsilon^{*}(x)\right] x \mathrm{~d} x=0
\end{aligned}
$$

leading to the following relationships

$$
\begin{aligned}
& \frac{\left(x_{\mathrm{R}}+x_{\mathrm{L}}\right) b}{2}+a\left(x_{\mathrm{R}}-x_{\mathrm{L}}\right)-\left(x_{\mathrm{R}}-x_{\mathrm{L}}\right) \Gamma=0 \\
& \frac{\left(x_{\mathrm{R}}^{2}+x_{\mathrm{R}} x_{\mathrm{L}}+x_{\mathrm{L}}^{2}\right) b}{3}+\frac{\left(x_{\mathrm{R}}^{2}-x_{\mathrm{L}}^{2}\right) a}{2} \\
& -\left(x_{\mathrm{R}}-x_{\mathrm{L}}\right)^{2} \Gamma_{1}=0
\end{aligned}
$$

Expressions are given explicitly in terms of the beam boundaries $x_{\mathrm{L}}$ and $x_{\mathrm{R}}$ for the purposes of generality, e.g. to allow the consideration of effects of surface layer removal.

The solution of the linear system for parameters $a$ and $b$ has the form

$$
\begin{aligned}
& a=\frac{6 \Gamma_{1}\left(x_{\mathrm{R}}^{2}-x_{\mathrm{L}}^{2}\right)-4 \Gamma\left(x_{\mathrm{R}}^{2}+x_{\mathrm{R}} x_{\mathrm{L}}+x_{\mathrm{L}}^{2}\right)}{\left(x_{\mathrm{R}}-x_{\mathrm{L}}\right)^{2}} \\
& b=\frac{12 \Gamma_{1}\left(x_{\mathrm{R}}-x_{\mathrm{L}}\right)-6 \Gamma\left(x_{\mathrm{R}}+x_{\mathrm{L}}\right)}{\left(x_{\mathrm{R}}-x_{\mathrm{L}}\right)}
\end{aligned}
$$

Noting that since the bending component of strain in terms of the beam bending radius $R$ and the beam curvature $\kappa$ is given by

$$
e=\frac{x}{R}=x \kappa
$$

then from equation (8) the curvature of the bent beam is found as

$$
\kappa=\frac{a}{h}=\frac{6 \Gamma_{1}\left(x_{\mathrm{R}}^{2}-x_{\mathrm{L}}^{2}\right)-4 \Gamma\left(x_{\mathrm{R}}^{2}+x_{\mathrm{R}} x_{\mathrm{L}}+x_{\mathrm{L}}^{2}\right)}{\left(x_{\mathrm{R}}-x_{\mathrm{L}}\right)^{3}}
$$

Equation (11) contains an expression that is useful for the analysis of beam curvature as a function of the eigenstrain distribution $\varepsilon^{*}(x)$.

Substituting equations (8) and (9) back into equation (2) gives the resulting prediction for the residual elastic strain distribution in the form

$$
\begin{aligned}
e(x)= & \frac{1}{\left(x_{\mathrm{R}}-x_{\mathrm{L}}\right)^{2}} \\
& \times\left\{6 \Gamma_{1}\left(x_{\mathrm{R}}-x_{\mathrm{L}}\right)\left(2 x-x_{\mathrm{R}}-x_{\mathrm{L}}\right)\right. \\
& \left.\quad+2 \Gamma\left[\left(x_{\mathrm{R}}^{2}+x_{\mathrm{R}} x_{\mathrm{L}}+x_{\mathrm{L}}^{2}\right)-3 x\left(x_{\mathrm{R}}+x_{\mathrm{L}}\right)\right]\right\} \\
& -\varepsilon^{*}(x)
\end{aligned}
$$

Equation (12) establishes the solution of the direct problem about the determination of residual elastic strain for an arbitrary given distribution of eigenstrain.

\section{INVERSE PROBLEM: DETERMINATION OF EIGENSTRAIN DISTRIBUTION FROM THE MEASURED RESIDUAL ELASTIC STRAIN}

The problem that will now be addressed in the present section stands in an inverse relationship to the one solved in the previous section. In practice, it is the residual elastic strain distribution that may be known, e.g. from diffraction measurement. Alternatively, changes in the elastic strain values can be monitored, e.g. using strain gauges, in the course of material removal, and the underlying eigenstrain distribution needs to be determined.

In practice, the residual elastic strain, or its increments, can only be measured at a finite number of points. Therefore the reconstruction of an unknown functional distribution is sought, i.e. an object with an infinite number of degrees of freedom, using a finite data set. Several difficulties may arise in this situation; e.g. whether the problem described in the previous section can be inverted; whether the inverse problem is regular, i.e. varies in a smooth fashion depending on the data; and whether the obtained solution is unique. In the present study an attempt is not made to answer these questions. Instead, an efficient inversion procedure is offered, leaving the evaluation of its uniqueness and regularity for future consideration.

Consider a set of experimental data consisting of the values of residual elastic strain (RES) $y_{j}$ collected 
at positions $x_{j}, j=1, \ldots, m$. In the present study it is assumed that the data were collected from a onedimensional scan in coordinate $x$. It is worth noting, however, that the approach presented below is not in any way limited to one-dimensional problems, and can be readily generalized to two- and threedimensional cases.

Denote by $e(x)$, as in the previous section, the predicted, or modelled residual elastic strain distribution. Evaluating $e(x)$ at each of the measurement points gives the predicted values $e_{j}=e\left(x_{j}\right)$. In order to measure the goodness of the prediction a functional $J$ is formed, given by the sum of squares of differences between actual measurements and the predicted values, with weights

$$
J=\sum_{j=1}^{m} w_{j}\left(y_{j}-e_{j}\right)^{2}
$$

The choice of weights $w_{j}$ is left to the modeller; e.g. they could be chosen based on the accuracy of measurements being interpreted. Minimization of the functional $J$ provides a rational variational basis for selecting the most suitable model to match the measurements, in terms of the overall goodness of fit.

It will now be assumed that the unknown eigenstrain distribution, yet to be determined, is given by a truncated series of basis distributions

$$
\varepsilon^{*}(x)=\sum_{i=1}^{N} c_{i} \xi_{i}(x)
$$

Here $N$ is the total number of basis distributions used in the prediction. The results of the previous section contain the analytical procedure for the solution of the direct problem, i.e. the determination of the residual elastic strain distribution that arises in response to an arbitrary eigenstrain distribution $\varepsilon^{*}(x)$. This procedure can now be applied to each of the $N$ basis distributions $\xi_{i}(x)$ in turn. As a result, a family of residual elastic strain solutions $E_{i}(x)$ is obtained.

Due to the linearity of the direct problem, the predicted values $e_{j}$ of the residual elastic strain due to the eigenstrain distribution $\varepsilon^{*}(x)$ of equation (14) can themselves be written in the form of a superposition of responses to the basis eigenstrain distributions

$$
e_{j}=\sum_{i=1}^{N} c_{i} E_{i}\left(x_{j}\right)=\sum_{i=1}^{N} c_{i} e_{i j}
$$

with the same coefficients $c_{i}$ as in equation (14).

The inverse problem of determining the unknown eigenstrain distribution $\varepsilon^{*}(x)$ has now been reduced to the problem of determination of $N$ unknown coefficients $c_{i}$, which deliver a minimum to the functional $J$ in equation (13) and may now be rewritten as

$$
J=\sum_{j=1}^{m} w_{j}\left(\sum_{i=1}^{N} c_{i} e_{i j}-y_{j}\right)^{2}
$$

The above expression is quadratic and positive definite in the unknown coefficients $c_{i}$. It follows that the functional has a unique minimum, found by satisfying the condition

$$
\nabla_{c} J=0 \quad \text { or } \quad \frac{\partial J}{\partial c_{i}}=0, \quad i=1, \ldots, N
$$

Due to the quadratic nature of the functional in equation (16), the system of equations in equation (17) is linear. Therefore, the solution for the unknown coefficients $c_{i}$ can be readily found without iteration by inverting the linear system arising in equation (17). This system is written out explicitly below.

The partial derivative of $J$ with respect to the coefficient $c_{i}$ can be written explicitly as

$$
\begin{aligned}
\frac{\partial J}{\partial c_{i}} & =2 \sum_{j=1}^{m} w_{j} e_{i j}\left(\sum_{k=1}^{N} c_{k} e_{k j}-y_{j}\right) \\
& =2\left(\sum_{k=1}^{N} c_{k} \sum_{j=1}^{m} w_{j} e_{i j} e_{k j}-\sum_{j=1}^{m} w_{j} e_{i j} y_{j}\right)=0
\end{aligned}
$$

For purposes of illustration, it will now be assumed that the weights are equal to unity, so that equation (18) simplifies to

$$
\frac{\partial J}{\partial c_{i}}=2\left(\sum_{k=1}^{N} c_{k} \sum_{j=1}^{m} e_{i j} e_{k j}-\sum_{j=1}^{m} e_{i j} y_{j}\right)=0
$$

The following matrix and vector notation are introduced

$$
\mathbf{E}=\left\{e_{i j}\right\}, \quad \boldsymbol{y}=\left\{y_{j}\right\}, \quad \boldsymbol{c}=\left\{c_{i}\right\}
$$

Noting that notation $e_{k j}$ corresponds to the transpose of matrix $\mathbf{E}$, the entities appearing in equation (19) can be written in matrix form as

$$
\mathbf{A}=\sum_{j=1}^{m} e_{i j} e_{k j}=\mathbf{E E}^{\mathrm{T}}, \quad \boldsymbol{b}=\sum_{j=1}^{m} e_{i j} y_{j}=\mathbf{E} \boldsymbol{y}
$$

Hence equation (19) assumes the form

$$
\nabla_{c} J=2(\mathbf{A} \boldsymbol{c}-\boldsymbol{b})=0
$$

The solution of the inverse problem has thus been reduced to the solution of the linear system

$$
\mathbf{A} \boldsymbol{c}=\boldsymbol{b}
$$

for the unknown vector of coefficients $\boldsymbol{c}=\left\{c_{i}\right\}$.

Whenever the solution of an inverse problem is sought, questions arise concerning the existence and uniqueness of the solution, and also concerning the 
well-posedness of the problem, i.e. the continuity of the dependence of the solution on the problem parameters, the choice of the basis functions, the number of terms $N$ in the truncated series, etc.

Within the present regularized formulation of the problem, for an arbitrary choice of the family of basis functions and arbitrary number of basis functions $N$, unique solution is guaranteed to exist. This is a consequence of the positive definiteness of the quadratic functional $J$. Furthermore, it is clear that increasing the number of terms $N$ is guaranteed to deliver a sequence of monotonically non-increasing values of $J$; i.e. the goodness of approximation will not be diminished.

An interesting question concerns the convergence of the solution, e.g. in terms of eigenstrain distribution $\varepsilon^{*}(x)$, to the 'true' solution, in the limit $N \rightarrow \infty$. Similarly, the continuity in the behaviour of the solution with the choice of basis functions deserves to be discussed. While it must be emphasized that these questions are clearly fundamental and ought to be addressed, the focus is currently placed on the development of a practical tool for residual strain analysis. Insofar as this is the aim of the present study, the proposed framework offers an efficient 'one shot' approach to the solution of the inverse problem. Furthermore, the choice of moderate values $N$, compared to the number of measurements, $m$, offers a rational procedure for smoothing the data, as discussed in the next section.

\section{APPLICATION TO THE ANALYSIS OF RESIDUAL ELASTIC STRAIN INDUCED BY LASER SHOCK PEENING}

The approach developed in the previous section is now applied to the problem of interpreting diffraction measurements of residual elastic strain in a slice parted off from a laser shock peened plate of an aerospace alloy Ti-6Al-4V [1] .

Figure 1 shows the geometry of the specimen. The $8.5 \mathrm{~mm}$ thick plate of titanium alloy was laser shock peened on the surface $x=0$, resulting in the creation of plastic strain in the near-surface regions and of the residual elastic strain that arose due to elastic equilibration. Instead of attempting to measure the residual stress or residual elastic strain in the complete plate, a thin $(3 \mathrm{~mm})$ slice of material was parted off from the plate, as shown in the figure.

The 'slice' was mounted on the Euler cradle. Highenergy X-ray diffraction measurements were performed in transmission on this slice, by initially aligning the beam with the $z$ direction. The incident

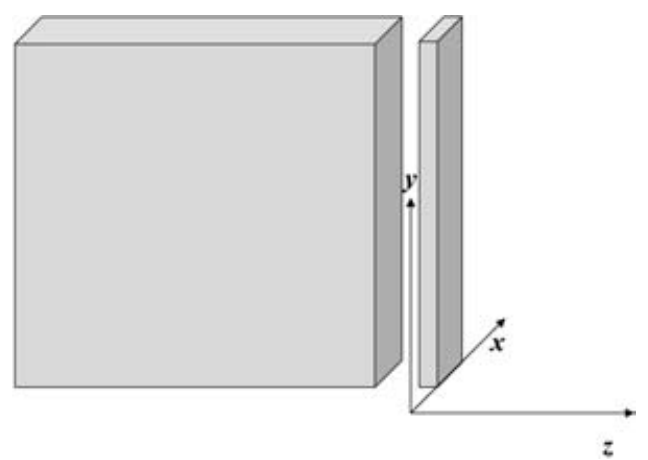

Fig. 1 Schematic illustration of the square coupon from the plate of Ti-64 alloy and the slice parted off for transmission diffraction investigation

beam spot was set using collimating slits to the size of $s_{x}=25 \mu \mathrm{m}$ by $s_{y}=100 \mu \mathrm{m}$. The beam was monochromated using a single bounce bent Laue crystal, which selected the photons with an energy of approximately $60 \mathrm{keV}$. The measurements of residual elastic strain were performed in the coupled $\theta-2 \theta$ scan configuration, thus ensuring that the $y$ direction within the sample (Fig. 1) always remained aligned with the scattering vector (the bisector of the incident and scattered beam). The height of the 'slice' was adjusted so that the beam spot was located in the middle of the sample length in the $y$ direction. The 'slice' was scanned in the $x$ direction across the beam, with several diffraction peaks collected at each $x$ position. The same peaks were also collected from a diffracting volume located at the corner of the 'slice', which was assumed to be stress (and strain) free, and provided the reference peak positions for each of the reflections.

Figure 2(a) shows the profile of the residual elastic strain component $e_{y y}$ for reflections (00.2) and (11.0) parallel to the laser shock peened surface plotted as a function of distance along the $x$ direction from the treated surface across the entire sample depth. It is important to note the difference between the two profiles, arising from the anisotropy of deformation response of the individual hcp (hexagonal closepacked) crystals within the polycrystalline aggregate, both in the elastic and plastic regime. Elastic anisotropy gives rise to a difference in the strain experienced by the crystallites in response to the same applied stress, due to the variation of stiffness with the orientation within the crystal. Plastic anisotropy gives rise to a difference in the maximum strain that can be sustained within the crystal, since strain can be accommodated by plastic slip. This phenomenon does not give rise to a peak shift, and appears in the diffraction experiment as strain 'shedding'. The difference in the measured residual elastic strains in 


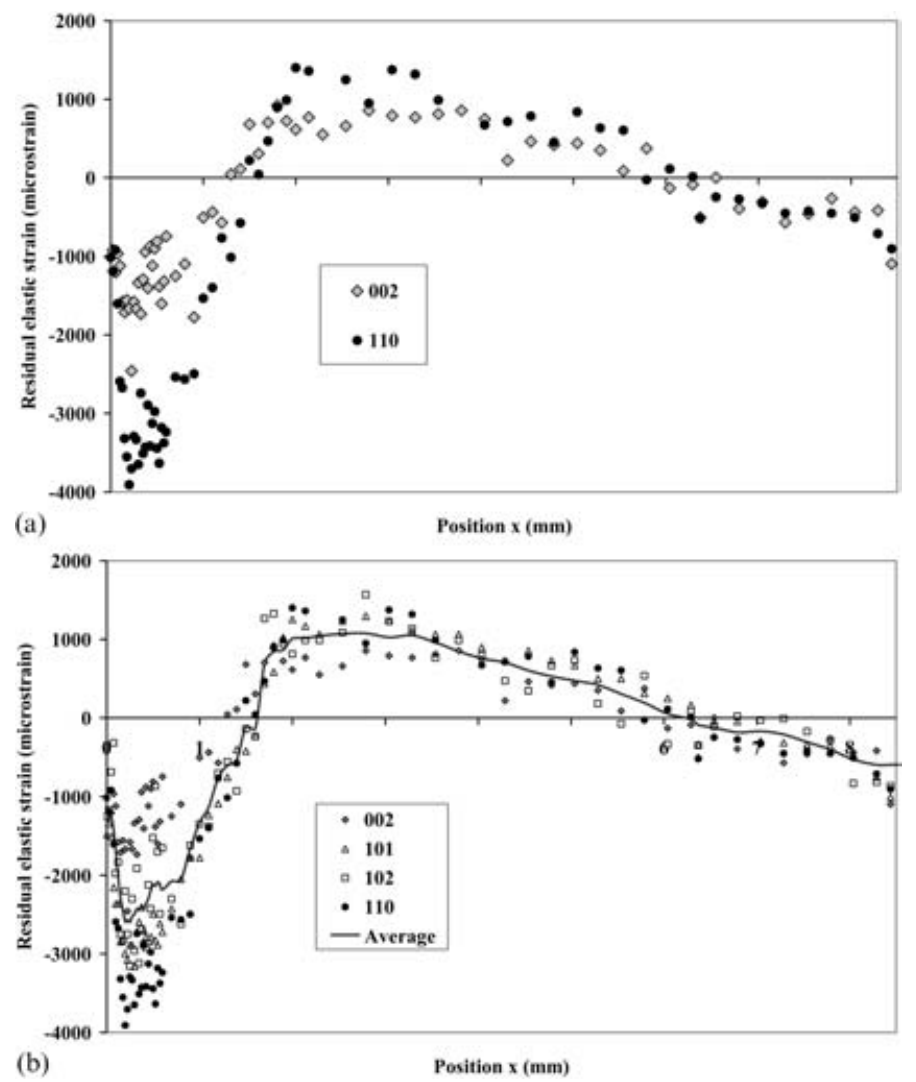

Fig. 2 (a). Profile of residual elastic strains for reflections (00.2) and (11.0) parallel to the laser shock peened surface plotted as a function of distance from the treated surface across the entire sample depth. (b). Profile of residual elastic strains parallel to the laser shock peened surface plotted as a function of distance from the treated surface across the entire sample depth. Markers indicate the strain values computed using individual diffraction peaks (00.2), (10.1), (10.2), and (11.0). The line indicates an estimate of the macroscopic average residual elastic strain computed using appropriate weighting of the available peak strains

the plastically deformed region therefore primarily reflects the anisotropy of the ease with which plastic slip can take place in different orientations and is related to the macroscopic plastic strain (see below). The difference in the measured residual elastic strains in the elastic region primarily reflects the anisotropy of Young's modulus (stiffness) with the crystallites.

Figure 2(b) shows further profiles of the residual elastic strain component $e_{y y}$ parallel to the laser shock peened surface plotted as a function of distance along the $x$ direction from the treated surface across the entire sample depth. Markers indicate the strain values computed using individual diffraction peaks (00.2), (10.1), (10.2), and (11.0). The line indicates an estimate of the macroscopic average residual elastic strain computed using appropriate weighting of the available peak strains [6, 7].

Figure 3 shows the plot of the 'difference' strain plotted as a function of depth from the laser shock surface, obtained by subtracting the (11.0) strain values from the (00.2) stain values. High values of the 'difference strain' indicate the region where significant plastic deformation took place. Also plotted (cross markers) is the plastic strain, computed as the difference between the measured residual elastic strain profile and the 'pure bending' line, and multiplied by a factor of 0.5 for ease of comparison.

The following assumptions were made for the purposes of interpretation of the measurements.

1. It was assumed that parting off the 'slice', which was performed using slow electrical discharge machining (EDM), did not cause the eigenstrain distribution within the piece to be modified. At any rate, it may be stated with reasonable confidence that any additional deformation or residual stress generation due to spark erosion (EDM) can be expected to be confined to a very shallow layer close to the newly created surface; i.e. it did not extend deeper than about $100 \mu \mathrm{m}$ in the $z$ direction from the newly created surface. 


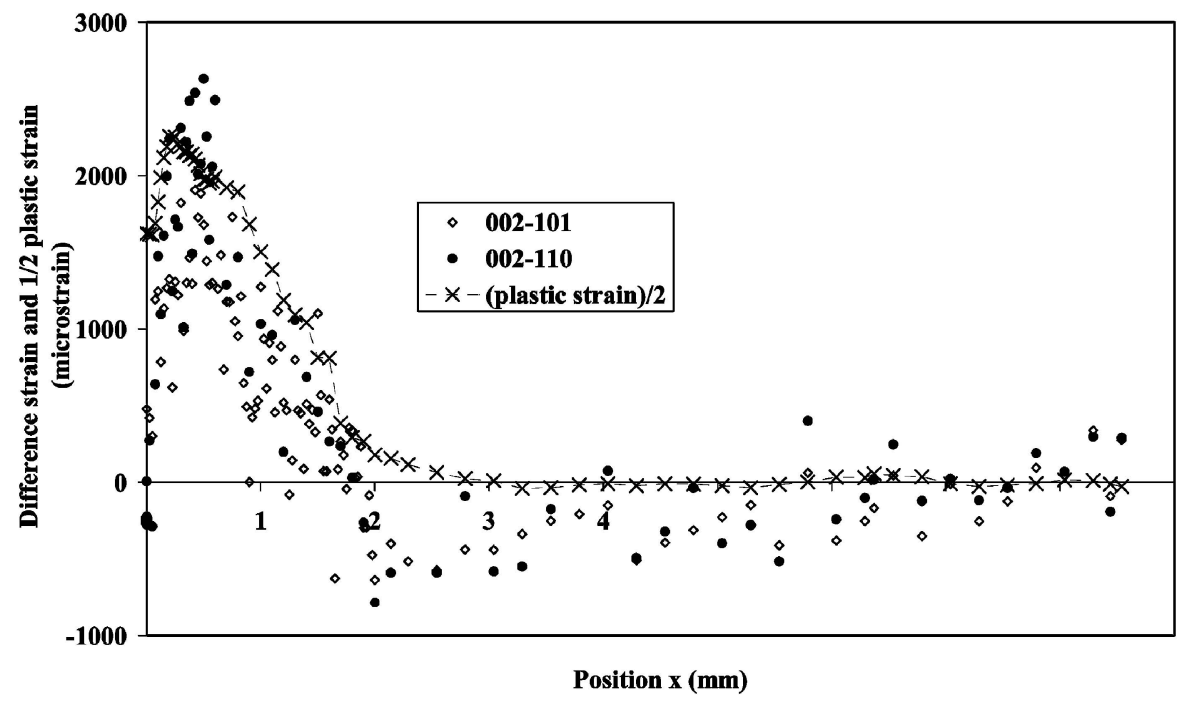

Fig. 3 'Difference strain' plotted as a function of depth from the laser shock surface. High values of the 'difference strain' indicate the region where significant plastic deformation took place. Also plotted (cross markers) is the plastic strain, worked out as the difference between the measured residual elastic strain profile and the 'pure bending' line, and multiplied by a factor of 0.5 for ease of comparison

2. It was further ensured that the scattering volume (gauge volume) was completely embedded within the slice, so that near-surface effects did not contribute to the measured peak profile.

3. It was assumed that the redistribution and relaxation of the residual elastic strain within the 'slice' primarily concerned the $z$ component and that the effect of the eigenstrain component $\varepsilon_{z z}^{*}$ can be neglected in the analysis. This assumption can be made on the basis of the finite element investigation of the residual elastic strain response to eigenstrain distributions [8].

4. It was assumed that the only eigenstrain component relevant to the analysis of the residual elastic strain state within the slice is the component $\varepsilon_{y y}^{*}$ and that the analysis of residual elastic strain can be conducted within the approximation afforded by the Kirchhoff beam bending theory.

The depth of the eigenstrain distribution, $x_{0}$, was considered to be an external parameter set at the beginning of analysis, and not adjusted in the process. The reason for this choice is that, in the present approach, the dependence of the functional $J$ on this parameter is non-linear and the value of the distribution depth, $x_{0}$, cannot be obtained from the single inversion of the linear system. It is possible, however, to devise an iterative procedure for establishing the best value of $x_{0}$, e.g. by comparing the 'goodness of fit', i.e. the values of the functional $J$ obtained taking different values of $x_{0}$.
In the present study the value of $x_{0}$ was assumed to be equal to $2.3 \mathrm{~mm}$. Although in principle this parameter may also be refined, the necessary nonlinear optimization procedure was not implemented since it requires a distinctly different set of tools. In practice, an approximate determination of the eigenstrain distribution depth was carried out by fitting a straight line to the elastically bent part of the beam and registering the point of significant deviation of the residual elastic strain profile from this line.

It is important to note that, provided the assumed depth of eigenstrain distribution exceeds a minimum value, the variational procedure adopted here is capable of describing the smooth decay of the eigenstrain distribution to a zero value that it assumes on part of the domain of its existence. A rational approach may be adopted whereby the domain of existence of eigenstrain coincides with the entire width of the beam. The most appropriate choice of basis functions in this case will, however, be different from that used here.

The system of basis functions considered in the present study was of the form

$$
\xi_{i}(x)=\left(x-x_{0}\right)^{i+2}, \quad i=1, \ldots, 6
$$

This choice of the functional basis ensures that at $x=x_{0}$ both the eigenstrain and its derivative turn to zero, thus giving rise to the prediction of a smooth residual elastic strain profile. 
Figure 4 shows the comparison between the measured RES shown by the markers and the eigenstrain model prediction, shown by the continuous curve. The predicted RES profile was computed following the procedures described in the present paper.

\section{RESULTS AND DISCUSSION}

The agreement between the predicted and measured profiles in Fig. 4 is excellent, perhaps with the exception of the region close to the edge of the eigenstrain distribution, around $x=2.5 \mathrm{~mm}$. Increasing the number of terms in the functional basis [equation (24) ] or modifying the depth of the eigenstrain distribution, $x_{0}$, does not lead to an improvement in the quality of fit. Various explanations can be offered for the observed phenomenon. One possibility is that in this region relatively small values of plastic strain are observed. In a polycrystalline material this is likely to lead to some grains sharing a favourable orientation for plastic slip undergoing yielding, while other grains remain elastic. This leads to additional deviations in elastic strains measured for certain reflections, so that the interpretation of diffraction data in terms of the average macroscopic RES may become particularly challenging.

Figure 5 shows the comparison between two methods of evaluating eigenstrain. Markers show the eigenstrain profile obtained from direct interpretation of the measured data, by subtracting the straight line fit to the pure bending part of the RES profile (see Fig. 4). This is the same method of interpretation used to obtain information about the plastic strain distribution. Indeed, in the present context plastic strain and eigenstrain are terms that can be used interchangeably, although a caveat must be made that this does not apply in all situations. A continuous curve shows the eigenstrain model prediction obtained using the procedure described in the present paper, i.e. global minimization of the sum of squared differences between predicted and measured RES values at measurement points.

The agreement between the two methods of eigenstrain evaluation in Fig. 5 is clearly good, but not perfect. This situation is likely to arise due to the difference in the definition of eigenstrain: in the present context it refers to the equivalent average macroscopic plastic strain, while in diffraction analysis it may be approximately determined using the difference strain, i.e. the mismatch between residual elastic strains calculated from different reflections. The correlation of the latter parameter with plastic strain has been established, but it does not amount to simple equality. Furthermore, the use of independent reference peak positions for each reflection in the present set-up is likely to give rise to small inconsistencies that affect the RES magnitudes, and ultimately the strain profile across

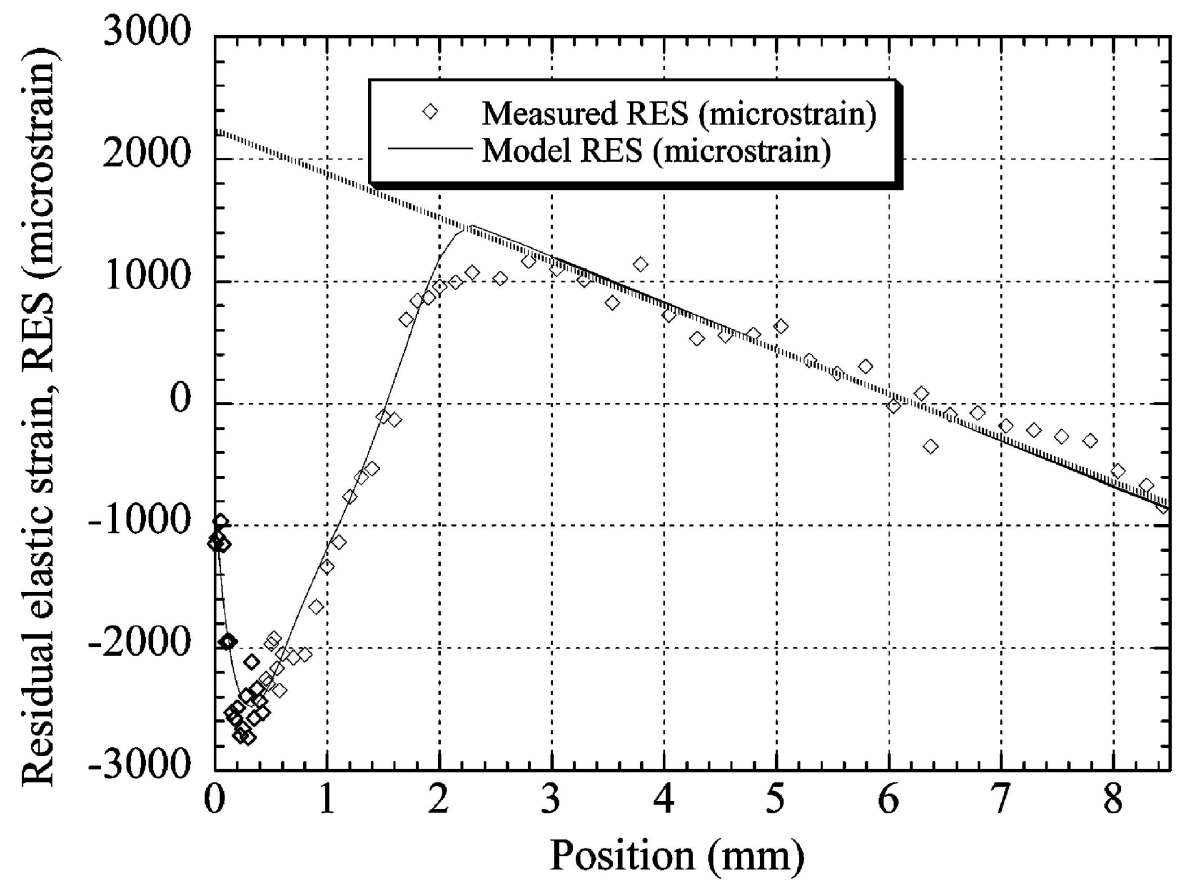

Fig. 4 Comparison between measured residual elastic strain (RES) shown by the markers and the eigenstrain model prediction shown by the continuous curve 


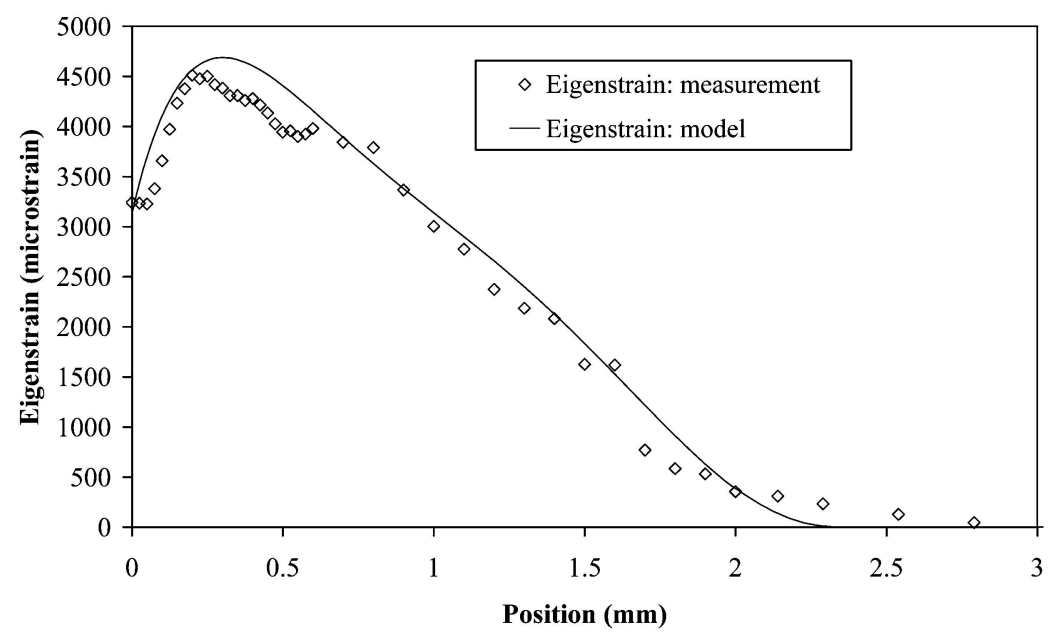

Fig. 5 Comparison between two methods of evaluating eigenstrain. Markers show the eigenstrain profile obtained from direct interpretation of the measured data by subtracting the straight line fit to the pure bending part of the residual elastic strain profile (see Fig. 4). The continuous curve shows the eigenstrain model prediction obtained using the procedure described in the present paper

the slice. An experimental approach that allows these difficulties to be overcome involves the use of energy dispersive X-ray diffraction coupled to Rietveld refinement of the complete collected diffraction profile $[\mathbf{9}, \mathbf{1 0}]$.

The procedure put forward in the present paper provides a rational algorithm for the evaluation of the most likely underlying eigenstrain distribution responsible for the entire set of measurements available for interpretation. A judicious choice must be made for the order of approximation, $N$, given by the number of functions in the basis [equation (24)]. Increasing $N$ unduly amplifies the oscillatory behaviour of the predicted solution, leading to approximate profiles containing the level of detail that cannot be justified with any confidence. On the other hand, selecting a moderate value of $N$ provides a natural method of smoothing out inherently 'noisy' strain measurement data.

It is interesting to note that the analysis of residual elastic strains arising due to eigenstrains in a beam does not require the knowledge of material's stiffness, although it uses the assumption of a linear relationship between elastic strains and stresses.

It is appropriate here to provide an outline of the way in which the proposed approach serves as the basis for the reconstruction of residual stress. Plastic deformation arising as a consequence of the laser shock peening process considered in the present example was isotropic within the planes parallel to the surface of the peened plate. It is logical to draw the conclusion that the eigenstrain distribution within the plate that arose as a consequence of this process (at least remotely from plate edges) must also be isotropic. Hence the residual stress state $\sigma$ within the complete plate prior to separating off the slice is equi-biaxial within every plane parallel to the peened surface, and is related to the biaxial residual elastic strain $e$ via

$$
\sigma=\frac{E}{1-v} e
$$

where $E$ is Young's modulus and $v$ is Poisson's ratio. In comparison, when the parted-off slice of small width is being considered, then the effect of the transverse component of peening-induced eigenstrain can be ignored, and the residual elastic strain state can be thought of as uniaxial, i.e. in the beam bending approximation

$$
\sigma=E e
$$

It is very interesting to note that the predicted residual elastic strain profile is unchanged between the two cases. This observation, made here on the basis of beam and plate approximations, was verified and confirmed through numerical experiments using finite element modelling [8]. Thus, the separation of the slice results in relaxation of residual stress of the order of 30 per cent for most metallic materials. It is also important to note that no additional plastic deformation occurs during this process, i.e. the underlying eigenstrain distribution remains unchanged.

The present application of the paradigm was cast in the context of beam bending theory, since it 
provides a convenient vehicle for demonstrating the flexibility and power of the proposed approach. It is important to note, though, that the method possesses a very considerable degree of flexibility, and can be applied with equal success within the framework of other numerical modelling tools.

\section{REFERENCES}

1 Korsunsky, A. M., Liu, J., Laundy, D., Golshan, M., and Kim, K. Residual elastic strain due to laser shock peening: synchrotron diffraction measurement, J. Strain Analysis, 2006, 41(2), 113-120.

2 Mura, T. Micromechanics of defects in solids, 1987 (Martinus Nijhoff Publishers, Dordrecht, The Netherlands).

3 Ueda, Y. and Yuan, M. G. Prediction of residual stresses in butt welded plates using inherent strains. J. Engng Mater. Technol., 1993, 115, 417-423.

4 Prime, M. B. and Hill, M. R. Measurement of fiberscale residual stress variation in a metal-matrix composite. J. Composite Mater., 2004, 38(23), 2079-2095.
5 Korsunsky, A. M. On the modelling of residual stresses due to surface peening using eigenstrain distributions, J. Strain Analysis, 2005, 40, 817-824.

6 Korsunsky, A. M., Daymond, M. R., and James, K. E. The correlation between plastic strain and anisotropy strain in aluminium alloy polycrystals. Mater. Sci. Engng A - Struct. Mater. Properties, Microstruct. and Processing, 2002, 334, 41.

7 Korsunsky, A. M., James, K. E., and Daymond, M. R. Intergranular stresses in polycrystalline fatigue: diffraction measurement and self-consistent modelling, Engng Fract. Mechanics, 2004, 71, 805-812.

8 Korsunsky, A. M., Regino, G. M., Nowell, D., and Liu, J. Synchrotron measurement and eigenstrain modelling of residual stress in machined nickel alloy plates. J. Strain Analysis, (submitted for publication).

9 Korsunsky, A. M., Collins, S. P., Owen, R. A., Daymond, M. R., Achtioui, S., and James, K. E. Fast residual stress mapping using energy-dispersive synchrotron X-ray diffraction on station 16.3 at the SRS. J. Synchrotron Radiat., 2002, 9, 77-81.

10 Liu, J., Kim, K., Golshan, M., Laundy, D., and Korsunsky, A. M. Energy calibration and fullpattern refinement for strain analysis using energydispersive and monochromatic X-ray diffraction. J. Appl. Crystallography, 2005, 38, 661-667. 